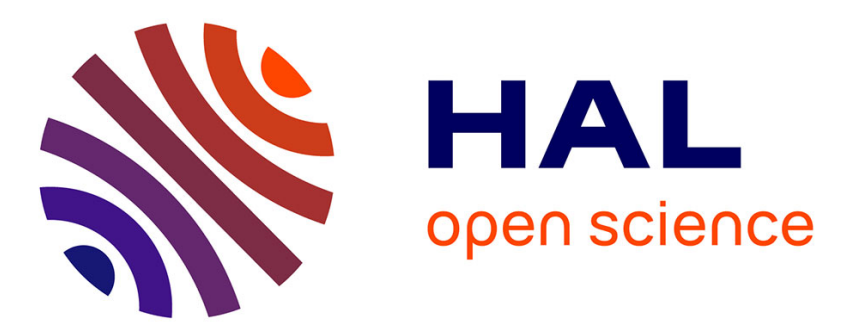

\title{
On the generation of octave-spanning optical frequency combs using monolithic whispering-gallery-mode microresonators
}

\author{
Y.K. Chembo, N. Yu
}

\section{- To cite this version:}

Y.K. Chembo, N. Yu. On the generation of octave-spanning optical frequency combs using monolithic whispering-gallery-mode microresonators. Optics Letters, 2010, 35 (16), pp.2696-2698. 10.1364/OL.35.002696 . hal-00581731

\section{HAL Id: hal-00581731 \\ https://hal.science/hal-00581731}

Submitted on 18 Apr 2021

HAL is a multi-disciplinary open access archive for the deposit and dissemination of scientific research documents, whether they are published or not. The documents may come from teaching and research institutions in France or abroad, or from public or private research centers.
L'archive ouverte pluridisciplinaire HAL, est destinée au dépôt et à la diffusion de documents scientifiques de niveau recherche, publiés ou non, émanant des établissements d'enseignement et de recherche français ou étrangers, des laboratoires publics ou privés. 


\title{
On the generation of octave-spanning optical frequency combs using monolithic whispering-gallery-mode microresonators
}

\author{
Yanne K. Chembo ${ }^{1,2, *}$ and Nan $\mathbf{Y u}^{1}$ \\ ${ }^{1}$ Jet Propulsion Laboratory, California Institute of Technology, 4800 Oak Grove Drive, Pasadena, California 91109, USA \\ ${ }^{2}$ Current address: Optics Department, FEMTO-ST Institute (UMR CNRS 6174), 16 Route de Gray, 25030 Besançon, France \\ ${ }^{*}$ Corresponding author: yanne.chembo@jpl.nasa.gov
}

\begin{abstract}
Octave-spanning optical frequency combs are especially interesting in optical metrology owing to the ability of selfreferencing. We report a theoretical study on the generation of octave-spanning combs in the whispering gallery modes of a microresonator. Through a modal expansion model simulation in a calcium fluoride microcavity, we show that a combination of suitable pump power, Kerr nonlinearity, and dispersion profile can lead to stable and robust octave-spanning optical frequency combs.
\end{abstract}

Optical frequency combs are sets of equidistant and extremely narrow spectral lines in the UV, visible, or IR ranges [1-3]. They are typically generated using ultrafast mode-locked lasers in combination with highly nonlinear photonic crystal fibers. Recently it has been shown that they can also be generated using high- $Q$ whisperinggallery-mode (WGM) resonators [4-6]. In this case, photons are resonantly injected into the resonator and strongly confined into the toruslike WGMs. The ultrahigh $Q$-factor leads to long photon storage times, which enhances the Kerr nonlinearity of the microresonator bulk medium. Various cavity eigenmodes can, therefore, be excited through four-wave mixing (FWM) as the photons sequentially cascade from the pump to the other WGMs. In comparison with their mode-locked lasers counterparts, WGM optical frequency comb generators are characterized by a significantly reduced size and power consumption, along with a high repetition rate. They are, therefore, particularly interesting to miniaturization, chip integration, and space applications.

For purposes of self-referencing in optical metrology, it is important that the comb spans over at least one octave, as it allows a phase-coherent link between the optical carrier frequency and the comb spacing rf [1-3]. While there were several experimental demonstrations of comb generation, there is only one reported observation of an octave-spanning comb [7]. At the theoretical level, the comprehensive study is difficult for two main reasons: first, there is no standard model for the comb dynamics in the literature, and second, numerical simulations over an octave spectral span of several hundreds of nanometers are computationally challenging. Interestingly, Agha et al. have developed a theoretical model of comb generation in microcavities based on the nonlinear Schrödinger equation [8], and they were able to generate a comb of a $100 \mathrm{~nm}$ wavelength span. In this Letter, we report the successful simulation of an octave-spanning optical frequency comb using a modal expansion approach. Our results strongly suggest that experimental generation of these combs can be obtained in similar WGM resonators.
We consider a calcium fluoride $\left(\mathrm{CaF}_{2}\right)$ microdisk obtained from a truncated microsphere of radius $a=35 \mu \mathrm{m}$ (FSR $=1 \mathrm{THz} ; \mathrm{FSR}$, free spectral range), as displayed in Fig. 1. The small radius of the disk is chosen mainly out of consideration of reasonable computational feasibility. The orthonormal eigenmodes of the spherical resonator $V$ depend on the degenerated angular eigennumber $\ell$ and on the polarization $p$ (TE or TM). The eigenmodes $\Upsilon_{\ell p}(\mathbf{r})$ of the cavity can be explicitly written as $\Upsilon_{\ell, T E}(\mathbf{r}) \simeq$ $i \Upsilon_{\ell, \mathrm{TE}}(r, \theta, \phi) \mathbf{e}_{\theta}$ and $\Upsilon_{\ell, \mathrm{TM}}(\mathbf{r}) \simeq \Upsilon_{\ell, \mathrm{TM}}(r, \theta, \phi) \mathbf{e}_{r}$, with

$$
\Upsilon_{\ell p}(r, \theta, \phi)=\frac{(-1)^{\ell} \ell^{\frac{1}{4}}}{2^{\frac{1}{2}} \pi^{\frac{3}{4}}} \frac{S_{\ell p}(r) e^{-\frac{1}{2}\left(\theta-\frac{\pi}{2}\right)^{2}} e^{i \ell \phi}}{\sqrt{\int_{0}^{+\infty} S_{\ell p}^{2}(r) r^{2} d r}},
$$

where $S_{\ell p}$ is the radial profile of the WGMs [9], while $\mathbf{e}_{r}, \mathbf{e}_{\theta}$, and $\mathbf{e}_{\phi}$ are the orthonormal vectors in spherical coordinates. The electric field is expanded as $\mathbf{E}(\mathbf{r}, t)=$ $\sum_{\mu} \frac{1}{2} \mathcal{E}_{\mu}(t) e^{i \omega_{\mu} t} \Upsilon_{\mu}(\mathbf{r})+$ c.c., where c.c. stands for the complex conjugate, while $\mu \equiv\{\ell, p\}$ stands for the modes of slowly varying amplitude $\mathcal{E}_{\mu}(t)$ and frequency $\omega_{\mu}$. These eigenfrequencies are approximated by [10]

$$
\begin{aligned}
\omega_{\ell p}= & \frac{c}{n\left(\omega_{\ell p}\right) a}\left\{\left[\ell+\frac{1}{2}\right]+\xi_{1}\left[\frac{\ell+\frac{1}{2}}{2}\right]^{\frac{1}{3}}\right. \\
& \left.-\frac{p}{\sqrt{n_{0}^{2}-1}}+\frac{3}{20} \xi_{1}^{2}\left[\frac{\ell+\frac{1}{2}}{2}\right]^{-\frac{1}{3}}+\mathcal{O}\left[\ell+\frac{1}{2}\right]^{-\frac{2}{3}}\right\},
\end{aligned}
$$

where $p$ is equal to $n_{0} \equiv n\left(\omega_{0}\right)$ for a TE polarization and $1 / n_{0}$ for TM, while $\xi_{1}=2.338$ is the first root of the Airy

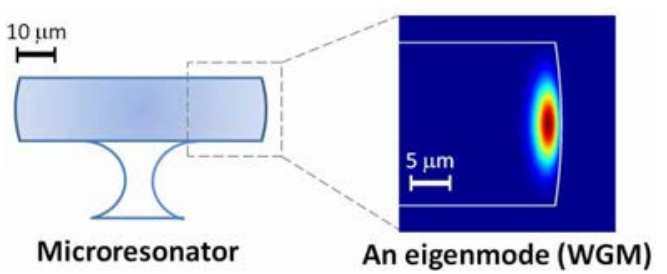

Fig. 1. (Color online) Lateral view of the microdisk resonator, and two-dimensional representation of the WGM $\ell_{0}=192$ (TE). 
function $\operatorname{Ai}(-z)$. These eigenfrequencies are weakly unequidistant because of the material and geometrical dispersions. One should note that the polar dependence of the eigenmodes is $\exp \left[-\frac{1}{2} \ell\left(\theta-\frac{\pi}{2}\right)^{2}\right]$, so the angular confinement of a WGM is of the order of $1 / \sqrt{\ell}$. As a consequence, the solutions of the microsphere are still valid for the truncated microdisk of Fig. 1 as long as the thickness $d$ of the microdisk is larger than the angular confinement. The wavelength $\lambda_{0}=2 \pi c / \Omega_{0}$ of the pump laser is finely tuned around $1556.8 \mathrm{~nm}$, corresponding to the TE mode of order $\ell_{0}=192$. The loaded quality factor of this resonantly coupled mode is set to $Q_{0}=10^{8}$.

We introduce the normalized electric field $\mathcal{A}_{\mu}=$ $\sqrt{\varepsilon_{0} n_{0}^{2} / 2 \hbar \omega_{\mu}} \mathcal{E}_{\mu}$, where $\left|\mathcal{A}_{\mu}\right|^{2}$ is the instantaneous photon number in the mode $\mu$, and $n_{0}$ is the real part of the refractive index at the laser frequency $\Omega_{0}$. The total electric field $\mathbf{E}(\mathbf{r}, t)$ obeys the Maxwell wave equation, with a complex, field- and frequency-dependent permittivity. After Hermitian inner-product projection onto the WGMs, the spatiotemporal wave equation is reduced to the following finite set of coupled rate equations:

$$
\begin{aligned}
\dot{\mathcal{A}}_{\eta}= & -\frac{1}{2} \Delta \omega_{\eta} \mathcal{A}_{\eta}+\frac{1}{2} \Delta \omega_{\eta} \delta_{\eta 0} \mathcal{F}_{0} e^{i \sigma t} \\
& -i g_{0} \sum_{\alpha, \beta, \mu} \Lambda_{\eta}^{\alpha \beta \mu} \mathcal{A}_{\alpha} \mathcal{A}_{\beta}^{*} \mathcal{A}_{\mu} e^{i \varpi_{\alpha \beta \mu \eta} t} .
\end{aligned}
$$

In this equation, $\Delta \omega_{\eta}=2 \Upsilon_{\eta} \omega_{\eta} n_{a} / n_{0}$ is the modal bandwidth, where $n_{a}$ is the imaginary part of the refraction index responsible for material absorption, and $\Gamma_{\eta}=$ $\int_{V}\left\|\Upsilon_{\eta}\right\|^{2} d V$ is the modal confinement factor. The FWM gain at the eigenfrequency $\omega_{0}$ of the mode $\ell_{0}$ is $g_{0}=$ $n_{2} c \hbar \omega_{0}^{2} / n_{0}^{2} V_{0}$, where $n_{2}$ is the nonlinear Kerr coefficient, and $V_{0}=\left[\int_{V}\left\|\Upsilon_{0}\right\|^{4} d V\right]^{-1}$ is the effective volume of the mode $\ell_{0}$. The intermodal coupling factor

$$
\Lambda_{\eta}^{\alpha \beta \mu}=\frac{\omega_{\mu}^{2}}{\omega_{0}^{2}} \sqrt{\frac{\omega_{\alpha} \omega_{\beta} \omega_{\mu}}{\omega_{\eta}^{3}}} \frac{\int_{V}\left[\mathbf{\Upsilon}_{\eta}^{*} \cdot \mathbf{\Upsilon}_{\mu}\right]\left[\mathbf{\Upsilon}_{\beta}^{*} \cdot \mathbf{\Upsilon}_{\alpha}\right] d V}{\int_{V}\left\|\mathbf{\Upsilon}_{0}\right\|^{4} d V}
$$

defines the coupling strength between the four interacting modes $\alpha, \beta, \mu$, and $\eta$, which depend on their power density overlap. As indicated by the Kronecker symbol $\delta_{\eta 0}$, the external field $\mathcal{F}_{0}$ is only resonant with the mode $\ell_{0}$, with a detuning $\sigma=\Omega_{0}-\omega_{0}$. The ideal resonance condition occurs when the modal FWM frequency detuning $\varpi_{\alpha \beta \mu \eta}=\omega_{\alpha}-\omega_{\beta}+\omega_{\mu}-\omega_{\eta}$ vanishes. It corresponds to the FWM interactions $\hbar \omega_{\alpha}+\hbar \omega_{\mu} \rightarrow \hbar \omega_{\beta}+\hbar \omega_{\eta}$ for which the energy and the total angular momentum of the interacting photons are conserved $\left(\ell_{\alpha}+\ell_{\mu}=\ell_{\beta}\right.$ $+\ell_{\eta}$ ). In a dispersionless cavity, the eigenmodes are perfectly equidistant and $\varpi_{\alpha \beta \mu \eta}=0$. We consider the case where the microresonator is pumped at resonance $(\sigma=0)$. The threshold number of intracavity photons needed for comb generation is equal to $\left|\mathcal{A}_{\mathrm{th}}\right|^{2}=$ $\Delta \omega_{0} / 2 g_{0}=n_{0}^{2} V_{0} / 2 \hbar \omega_{0} n_{2} c Q$, where $\Delta \omega_{0}$ is the bandwidth of the mode $\ell_{0}$.

We have simulated Eq. (3) numerically and studied the resulting comb spectrum. Once the geometry, the bulk medium, and the losses of the microresonator are given, the only free parameters of the system are the laser pump power $\left|\mathcal{F}_{0}\right|^{2}$ and its detuning frequency $\sigma$ relative to the WGM resonance. In particular, material dispersion is fixed by the frequency-dependent refraction index $n(\omega)$. To characterize the cavity dispersion [11], we use the material dispersion values tabulated by the Sellmeier expansion of [12]. The geometrical dispersion is determined by Eq. (2). The rate Eqs. (3) have been numerically integrated using the fourth-order Runge-Kutta algorithm. The cavity is pumped at the WGM of order $\ell_{0}=192$. We included 201 modes ranging from $\ell_{\min }=92$ to $\ell_{\max }=292$. The initial condition is vacuum ground states defined by $\left\langle\left|\mathcal{A}_{\ell}(0)\right|^{2}\right\rangle=\frac{1}{2}$, where the brackets stand for ensemble average over $\ell$.

Figure 2 displays two sample results of the numerical simulation. One of the most important parameters is the total photon number $\left|\mathcal{A}_{\text {in }}\right|^{2}$ inside the cavity. When the intracavity power is set 3 times above the threshold, the comb already spans beyond one octave [Fig. 2(a)]. The spectrum is, however, noticeably irregular and dominated by sparsely located spectral components. This strong spectral modulation indicates that the hyperparametric interactions are dominated by degenerate FWM. The degenerate interaction only generates pairwise comb lines that satisfy the required stability conditions. Nondegenerate FWM is responsible for filling the interstability regions and creating a more uniform comb line distribution [13]. In Fig. 2(b), the intracavity power is increased to 4 times the threshold value. The spectrum is now more uniform, thereby indicating that nondegenerate FWM interactions are much more strongly excited. We have also observed in our simulations that comb generation depends on the frequency detuning $\sigma$ and on the pump power.

In the simulation study, we also found that the use of strong pumping in this nonlinear system may lead to irregular oscillations in the temporal domain. Such oscillations lead to the emergence of parasitic modulation sidebands in the comb spectra (still within the bandwidth of the WGMs) [13]. For wide comb span generation,

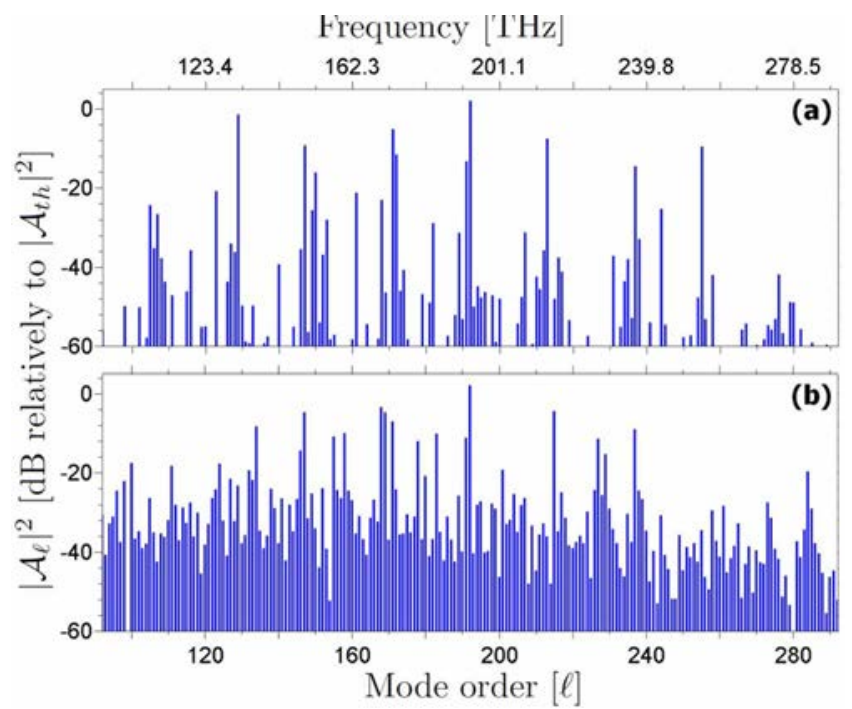

Fig. 2. (Color online) Numerical simulation of an octavespanning optical frequency comb. The intracavity power values are (a) $\left|\mathcal{A}_{\text {in }}\right|^{2}=3\left|\mathcal{A}_{\mathrm{th}}\right|^{2}$ and (b) $\left|\mathcal{A}_{\text {in }}\right|^{2}=4\left|\mathcal{A}_{\mathrm{th}}\right|^{2}$. 
therefore, one should have sufficient power to excite as many WGMs as possible, but not too much as to avoid these temporal instabilities. It is also noteworthy to point out that the simulation is computationally intensive. In the resonator, there is a huge number of FWM interactions of the kind $\Lambda_{\eta}^{\alpha \beta \mu} \mathcal{A}_{\alpha} \mathcal{A}_{\beta}^{*} \mathcal{A}_{\mu} e^{i \varpi_{\alpha \beta \mu \eta} t}$. All of them have to be considered simultaneously. For example, when we simulate $N$ modes (201 in our case), there are approximately $\frac{4}{3} N^{2} \sim 5 \times 10^{6}$ interactions.

In conclusion, we have carried out a simulation study on octave-spanning optical frequency comb generation with monolithic WGM microresonators. Our results show that, from a dynamical point of view, and as far as the dispersion and nonlinearity are concerned, it is possible to generate octave-spanning combs in WGM resonators. The choice of a small resonator was mainly driven by the consideration of the numbers of modes needed in the simulation and the corresponding complexity of it. Our results suggest that octave-spanning combs should be possible in resonators of smaller FSRs. However, smaller FSRs mean more comb lines in a given span. A comb of FSR in tens of gigahertz would already contain several thousand WGMs. It is expected that the required pump power will increase accordingly. It is still an open question whether the higher pump power will induce temporal instability before the octave span is achieved in this case. It is also well known that self-phase modulation locally modifies the refraction index and, thereby, the mode profiles of WGMs. This effect is generally referred to as Kerr lensing. It could play an important role in comb generation when the microcavity is strongly pumped above threshold. Temperature stability may be another important technical issue. In fact, the strong confinement of WGMs in small volumes generates thermal lensing that affects the various WGM characteristics and, hence, the comb generation. Future work should also consider stochastic thermal fluctuations with emphasis on their effects on the phase noise performances of the comb lines.

This work was performed at the Jet Propulsion Laboratory (JPL), California Institute of Technology, under a contract with NASA. Yanne K. Chembo acknowledges a fellowship from the NASA Postdoctoral Program, administered by Oak Ridge Associated Universities. Authors also acknowledge logistic support from the JPL Supercomputing and Visualization Facility.

\section{References}

1. S. T. Cundiff and J. Ye, Rev. Mod. Phys. 75, 325 (2003).

2. J. L. Hall, Rev. Mod. Phys. 78, 1279 (2006).

3. T. W. Hänsch, Rev. Mod. Phys. 78, 1297 (2006).

4. P. Del'Haye, A. Schliesser, O. Arcizet, T. Wilken, R. Holzwarth, and T. J. Kippenberg, Nature 450, 1214 (2007).

5. A. A. Savchenkov, A. B. Matsko, V. S. Ilchenko, I. Solomatine, D. Seidel, and L. Maleki, Phys. Rev. Lett. 101, 093902 (2008).

6. I. S. Grudinin, N. Yu, and L. Maleki, Opt. Lett. 34, 878 (2009).

7. P. Del'Haye, T. Herr, E. Gavartin, R. Holzwarth, and T. J. Kippenberg, "Octave spanning frequency comb on a chip," arXiv:0912.4890v1 (2009).

8. I. H. Agha, Y. Okawachi, and A. L. Gaeta, Opt. Express 17, 16209 (2009).

9. B. R. Johnson, J. Opt. Soc. Am. A 10, 343 (1993).

10. S. Schiller, Appl. Opt. 32, 2181 (1993).

11. P. Del'Haye, O. Arcizet, M. L. Gorodetsky, R. Holzwarth, and T. J. Kippenberg, Nat. Photon. 3, 529 (2009).

12. M. Daimon and A. Masumura, Appl. Opt. 41, 5275 (2002).

13. Y. K. Chembo, D. V. Strekalov, and N. Yu, Phys. Rev. Lett. 104, 103902 (2010). 